second stage of rehabilitation was supported by thermo-massotherapy. The results were superior to the first stage, the improvement of the clinical parameters was over $83,4 \%$.

The third therapeutic stage was applied 3-6 months later, after a new consultation. We used the same kinetic programme of strengthening- co-ordination exercises daily, for another two weeks, thermo-massotherapic supported.

The improvement after the third stage of the kinetic programme was superior, clinical parameters increased over $90 \%$ confronted with initial values.

Conclusion We prove the necessity of an unitary score of valuation and the efficiency of a staggered kinetic programme in the complex rehabilitation of the algo-functional muscular-ligamentar cervical syndromes, as the only efficient therapeutic alternative in the functional deficiencies? compensation and for prevention of complications.

\section{AB0167 EFFICIENCY OF REHABILITATION IN THE OLD PATIENTS PERIARTHRITIS}

EL Sidenco. Rehabilitation, University Hospital Bucharest, Bucharest, Romania

10.1136/annrheumdis-2001.584

\section{Background}

Objectives We studied a group of 40 old patients with soft tissues sufferings (periarthritis) at the shoulder or hip. Patients accused pain and limited mobility, and at the clinical examination we found muscular hypotrophy and limitation of function in the shoulder or hip.

Methods We applied an associated kinetic programme of stretching-strengthening- control and co-ordination exercises in two different periods of 15 days, supported by electro-thermo-massotherapy. The valuations were made at the beginning and the end of the programme, on a clinical score of valuation obtained by the clinical parameters; the results were statistically processing.

Results We find from the beginning a large incidence of the periarthritis at the old patients $(25,37 \%)$ and the preference for the women, larger in comparison with other ages ( 27 cases from the group of 40).

Application of the kinetic programme, physically supported, significantly improved the pain (completely for the spontaneous and nocturnal pain, and over $70 \%$ for the pain at the motion). Limitation of mobility increased over $63,6 \%$, even the functional remaining is limited at the old patient and the real possibilities of the kinetic programme display can be limited by the associated pathologies. Muscular hypotrophy had a medium response (improvement under 33,7\%), because of the limited biological capital and also the associated pathologies which imposed the adaptation of the kinetic programme to the real effort?s possibilities. Global function of the upper limb increased over 77,5\%.

Conclusion We consider the soft tissues sufferings of the old patient indicated for the kinetic programme, adequately supported by physical proceedings; the associated cardiovascular diseases do not forbid the rehabilitation, kinetic programme specially, if the patients are well medically treated and the kinetic programme is well adapted at the real effort?s possibilities of the patients.

\section{AB0168 PSYCHOPATHOLOGIC STUDY IN PATIENTS WITH FIBROMYALGIA}

${ }^{1}$ AR De la Serna, ${ }^{2} \mathrm{M}$ Serra, ${ }^{2} \mathrm{~J}$ Wulf. ${ }^{1}$ Rheumatology (1)/Psychosomatic (2); ${ }^{2}$ Psychosomatic, Sant Pau, Barcelona, Spain

10.1136/annrheumdis-2001.585

Background Fibromyalgia is considered a psychosomatic disease. Objectives This study was conducted to assess psychopathology changes in patients with fibromyalgia.

Methods We evaluated sequential outpatients with ACR criteria of fibromyalgia, through interviews by clinical psychologist, a psychiatrist and test for general psychopathology (Golberg), depression (Beck) and anxiety (Stai).

Results Demographic data: Twenty-nine patients were evaluated (4 men). Twenty-seven were married. Eighteen were immigrants. Twenty-four had children. Eight were unemployed. Thirteen were house wives. Three non qualified professionals. Eleven retailers and two qualified professionals. Psychopathologic results: Eight patients were totally normal. Golberg'test: twelve patients were normal. Five had mild and twelve severe alterations. Beck'test: thirteen patients were normal. Four suffered from mild depression. Four moderate, four severe and four more severe depression. Stai'test: ten patients didnt anxiety. Eighteen were trait anxiety and sixteen state anxiety. Interview: nineteen patients had sleep problems. Twenty-four were fatigued and fifteen had relapse thinking.

Conclusion The absence of psychopathology in $28 \%$ of patients with fibromyalgia, and the mild changes in others, allows us to differentiate between psychogenic rheumatism and fibromyalgia, and obligues us to establish a psychogenic evaluation in these.

\section{AB0169 FIBROMYALGIA. GERIATRICS PROBLEM?}

A Laiz, H Corominas, M Peiró, S Fernández, C Díaz, G Vázquez. Internal Medicine, Hospital de La Santa Creu I Sant Pau, Barcelona, Spain

10.1136/annrheumdis-2001.586

\section{Background}

Objectives To study in a group of patients, of a rheumatoloy unit in primary care the presence of fibromyalgia and tocompare the results between those with more and less than 65 years.

Methods From a rheumatoloy unit in primary care, in Barcelona, during one year, we selected the patients with fibromyalgia.

The diagnostic was done according with ACR criteria 1990.

Stadistical method used for comparisions was the chi- square test and correction of continuidad.

Results Visits performed were 1555. The mean was $61 \pm 15$ (Table 1).

Between them $(\mathrm{p}<0.05)$.

\begin{tabular}{llll}
\multicolumn{4}{l}{ Abstract AB0169 Table 1 } \\
\hline & Less than 65 & Equal or more than 65 & Total \\
\hline Without fibromyalgia & 777 & 660 & 1437 \\
With fibromyalgia & 93 & 25 & 118 \\
Total & 870 & 685 & 1555 \\
\hline
\end{tabular}

Conclusion The prevalence of fibromyalgia is similar to others studies. 